\title{
带有非线性扰动的抽象控制系统 的逼近能控性
}

\author{
周 鸿 兴 \\ (山东大学数学系, 济南)
}

1. 问题和假设 1978 年, J. Henry 首次研究了半线珄抛物型系统分布控制的整体能控 性问题 ${ }^{\text {(1]. }}$. 本文作者在文献 [2] 中得到了把有限维情形与无穷维情形统一起来的半线性抽象 控制系统通近能控性理论. 在文献 [3] 中又讨论了半线性热方程的一维控制问题. 本文是对 上述结果的推广.

考虑自反 Banach 空间 $X$ 中带有非线性扰动的抽象控制系统

$$
y^{\prime}(t)+A y(t)-\alpha F(y(t), u(t))+B u(t), \quad t_{0}<t<T .
$$

设系统 (1) 的初始状态为 $y\left(t_{0}\right)-\xi \in X$. 假设算子 $-A$ 是 $X$ 上强连续半群 $S(\cdot)$ 的生成算 子. $B \in \mathscr{L}[U \rightarrow X]$ 一全体胦另一自反 Banach 空间 $U$ 入 $X$ 的有界线性算子组成的萢范空 间. $F: X \times U \rightarrow X$ 是关于 $y$ 和 $u$ 满足一致 Lipschitz 条件的非线性函数:

$$
\begin{aligned}
& \left\|F\left(y_{1}, u_{1}\right)-F\left(y_{2}, u_{2}\right)\right\| \leqslant C_{1}\left\|y_{1}-y_{2}\right\|+C_{2}\left\|u_{1}-u_{2}\right\|_{U}, \\
& y_{1}, y_{2} \in X, u_{1}, u_{2} \in U .
\end{aligned}
$$

$a>0$ 是事先给定的常数. 此时对任意给定的初始状态 $\xi \in X$ 以及控制 $u(\cdot) \in L^{\prime}\left(t_{0}, T ; U\right)$ $(1 \leqslant p \leqslant \infty)$, 系统 (1) 定义了唯一解 (mild 解) $y(\cdot) \in C\left(\left[t_{0}, T\right] ; X\right)$, 并对任袖 $m_{3}, m_{2} \in$ $L^{\prime}\left(t_{0}, T ; U\right)$ 有

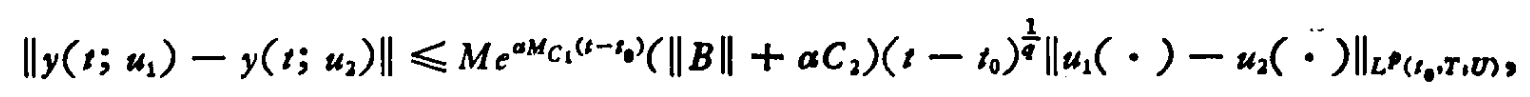

其中 $M=\max _{0<t<T}\|S(t)\|, \frac{1}{p}+\frac{1}{q}=1^{[2,4]}$.

定义算子 $\mathscr{S}_{\left(t_{0}, t_{1}\right)} \varphi-\int_{t_{0}}^{t_{1}} S\left(t_{1}-t\right) \varphi(t) d t, \varphi(\cdot) \in L^{p}\left(t_{0}, t_{1} ; X\right), t_{0}<t_{1} \leqslant T$, 以及

$$
(\mathscr{F} u)(\cdot)-F(y(\cdot ; u), u(\cdot)), u(\cdot) \in L^{p}\left(t_{0}, T ; U\right) \text {. }
$$

不难验证, 映 $L^{p}\left(t_{0}, T ; U\right) \lambda X$ 的算子 $\mathscr{S}_{\left(t_{0}, T\right)} B$ 以及 $\mathscr{S}_{\left(t_{0}, T\right)} \mathscr{T}$ 是连续的.

记系统 $(1)$ 的能达集为 $K_{\left(t_{0}, T\right)}(\xi ; \alpha F)-\left\{y(T ; u) \mid u(\cdot) \in L^{p}\left(t_{0}, T ; U\right)\right\}$, 如果对任意的 $\xi \in X$ 都有 $\overline{K_{\left(t_{0}, T\right)}(\xi ; \alpha F)}-X$, 则称系统 (1) 在 $\left[t_{0}, T\right]$ 上关于任意初始状态具有通近能控性 或简称系统 (1) 具有逼近能控性.

假设 (L).（i) $\mathscr{R}\left[S\left(T-t_{0}\right)\right] \subset \mathscr{R}\left[\mathscr{S}_{\left(t_{0}, T\right)} B\right](\mathscr{R}[\cdot]$ 表示算子的值域); (ii) 在 $X$ 中 存在赋范稠密线性子空间 $Z$; (iii) 对任意给定的 $\varepsilon>0$, 都可以定义一个映射 $\mathscr{V}_{8}: Z \rightarrow L^{p}\left(t_{0}\right.$, $T ; U)$, 使得 $\mathscr{V}_{\mathrm{s}} z \neq \varnothing, z \in Z$, 其中 $\mathscr{V}_{\mathrm{s}} z-\left\{v_{\mathrm{s}}(\cdot) \in L^{p}\left(t_{0}, T ; U\right) \mid\left\|z-\mathscr{S}_{\left(\mathrm{s}_{\mathrm{b}}, T\right)} B v_{\mathrm{s}}\right\|<\varepsilon\right.$,

本文 1983 年 2 月 1 日收到.

第 1 期

科学通报 
$\left.\left\|v_{\varepsilon}(\cdot)\right\|_{L^{p}\left(t_{0}, T_{Z}, U\right)} \leqslant k_{1}\left\|_{z}\right\|_{Z}\right\}, k_{1}>0$ 为某常数.

假设 (F). (i) $\mathscr{R}\left[\mathscr{S}_{\left(t_{0}, T\right)} \mathbb{Z}\right] \subset Z$; (ii) $\left\|\mathscr{S}_{\left(t_{0}, T\right)} \mathscr{F}_{u}\right\|_{Z} \leqslant k_{2}\|(\mathscr{F} u)(\cdot)\|_{L^{\left.P_{\left(f_{0}\right.}, T, X\right)}}$, $u(\cdot) \in L^{p}\left(t_{0}, T ; U\right), k_{2}>0$ 为某常数.

容易验证,文献 $[2,3]$ 中讨论的系统满足假设 (L)以及假设 $(F)$.

\section{2. 远近触控性}

定五 1 设系统(1)满足假设 (L)以及 (F), 则存在 $\alpha_{0}>0$, 只要 $0<\alpha<\alpha_{0}$, 系统 (1) 都 具有通近能控性.

证 设 $z \in Z, \xi \in X$ 以及 $0<\varepsilon<1$ 是任意给定的. $v_{1}(\cdot)$ 是 $L^{p}\left(t_{0}, T ; U\right)$ 中任一控 制,则由假设可知,对任何给定的 $\alpha>0$ 都存在

$$
\begin{aligned}
& v_{2} \in \mathscr{V}_{8}\left[z-S\left(T-t_{0}\right) \xi-\alpha \mathscr{S}_{\left(s_{0}, T\right)} \mathscr{I} v_{1}\right], \\
& w_{2} \in \mathscr{V}_{\varepsilon^{2}}\left[\alpha \mathscr{S}_{\left(t_{0}, T\right)} \mathscr{I} v_{1}-\alpha \mathscr{S}_{\left(t_{0}, T\right)} \mathscr{F} v_{2}\right] \text {. }
\end{aligned}
$$

记 $v_{3}(\cdot)-v_{2}(\cdot)+w_{2}(\cdot)$, 则有

$$
\begin{aligned}
\| z- & S\left(T-t_{0}\right) \xi-\alpha \mathscr{S}_{\left(t_{0}, T\right)} \mathscr{F} v_{2}-\mathscr{S}_{\left(t_{0}, T\right)} B v_{3} \| \\
& \leqslant\left\|z-S\left(T-t_{0}\right) \xi-\alpha \mathscr{S}_{\left(t_{0}, T\right)} \mathscr{F}_{v_{1}}-\mathscr{S}_{\left(t_{0}, T\right)} B v_{2}\right\| \\
& \quad+\left\|\alpha \mathscr{S}_{\left(s_{0}, T\right)} \mathscr{F}_{\nu_{1}}-\alpha \mathscr{S}_{\left(t_{0}, T\right)} \mathscr{F}_{v_{2}}-\mathscr{S}_{\left(t_{0}, T\right)} B w_{2}\right\| \\
& \leqslant \varepsilon+\delta^{2} .
\end{aligned}
$$

由归纳法可知, 存在序列 $\left\{v_{n}(\cdot) ; n-1,2, \cdots\right\} \subset L^{p}\left(t_{0}, T ; U\right)$, 它满足下面的

$$
\begin{gathered}
\left(v_{n+2}-v_{n+1}\right) \in \mathscr{V}_{\varepsilon^{n+1}}\left[\alpha \mathscr{S}_{\left(t_{0}, T\right)} v_{n}-\alpha \mathscr{S}_{\left(t_{0}, T\right)} v_{v_{n+1}}\right], \quad n-1,2, \cdots, \\
\left\|z-S\left(T-v_{0}\right) \xi-\alpha \mathscr{S}_{\left(v_{0}, T\right)} \mathscr{F} v_{n}-\mathscr{S}_{\left(t_{0}, r\right)} B v_{s+1}\right\| \leqslant \varepsilon+\varepsilon^{2}+\cdots+\varepsilon^{n}, \\
n-1,2, \cdots,
\end{gathered}
$$

记

$$
H\left(t_{0}, \alpha\right)-\alpha k_{1} k_{2}\left[M C_{1} e^{\alpha N C_{1}\left(T-t_{0}\right)}\left(\|B\|+\alpha C_{2}\right)\left(T-t_{0}\right)+C_{2}\right] .
$$

则利用(4)式以及(3)式可以得到

$$
\begin{aligned}
& \left\|v_{n+2}(\cdot)-v_{n+1}(\cdot)\right\|_{L} p_{\left(t_{0}, T, U\right)} \leqslant k_{1}\left\|\alpha \mathscr{S}_{\left(\delta_{0}, T\right)} \mathscr{v _ { n }}-a \mathscr{S}_{\left(t_{0}, T\right)} \mathscr{T} v_{n+1}\right\|_{2} \\
& \leqslant \alpha k_{1} k_{2}\left\|\mathscr{F} v_{n+1}-\mathscr{F}_{v_{n}}\right\|_{L^{p}\left(t_{0}, T, x\right)} \\
& \leqslant \alpha k_{1} k_{2}\left[C_{1} \| y\left(\cdot ; v_{n+1)}-y\left(\cdot ; v_{n}\right)\left\|_{L p_{\left(s_{0}, T, X\right)}}+C_{2}\right\| v_{n+1}(\cdot)\right.\right. \\
& \left.-v_{n}(\cdot) \|_{L^{p}\left(\varepsilon_{0}, T, U\right)}\right] \\
& \leqslant H\left(t_{0}, \alpha\right)\left\|_{v_{n+1}}(\cdot)-v_{n}(\cdot)\right\|_{L^{p}\left(t_{0}, T, U\right)}, \quad n=1,2, \cdots .
\end{aligned}
$$

对于任何事先给定的区间 $\left[r_{0}, T\right]$ 定义

$$
a_{0}-\min \left\{1 ; \frac{1}{k_{1} k_{2}}\left[M C_{1} e^{M C_{1}\left(T-t_{0}\right)}\left(\|B\|+C_{2}\right)\left(T-t_{0}\right)+C_{2}\right]^{-1}\right\} .
$$

于是, 只要 $0<\alpha<\alpha_{0}$, 由(6)式定义的 $H\left(t_{0}, \alpha\right)<1$, 因此满足(4)式的序列 $\left\{v_{n}(\cdot) ; n-1\right.$, $2, \cdots\}$ 是 $L^{p}\left(t_{0}, T ; U\right)$ 中的 Cauchy 序列. 于是存在 $\bar{\nu}(\cdot) \in L^{p}\left(t_{0}, T ; U\right)$, 使得在空间 $L^{p}\left(t_{0}, T ; U\right)$ 中有 $\lim _{m \rightarrow \infty} v_{*}(\cdot)-\bar{v}(\cdot)$. 根据算子 $\mathscr{S}_{\left(t_{0}, T\right)} B$ 以及 $\mathscr{S}_{\left(t_{0}, T\right)}$ 的强连续性以及 (5) 式我们有

$$
\left\|z-S\left(T-t_{0}\right) \xi-\alpha \mathscr{S}_{\left(t_{0}, T\right)} \mathscr{F} \bar{\nu}-\mathscr{S}_{\left(t_{0}, T\right)} B \bar{v}\right\| \leqslant \frac{\varepsilon}{1-\varepsilon} .
$$

由 $\xi \in X, 0<8<1$ 的任意性可知 $z \in \overline{K_{\left(t_{0}, T\right)}(\xi ; \alpha F)}$, 由 $z \in Z$ 的任意性可知

$$
Z \subset \overline{K_{\left(s_{0}, T\right)}(\xi ; a F)} \text {. }
$$


因为 $\mathrm{Z}$ 在 $X$ 中是稳密的, 所以 $\overline{K_{\left(t_{0}, T\right)}(\xi ; \alpha F)}-X$. 证毕.

假设 (L)' $\cdot$ (i)、(ii) 同假设 (L); (iii) $Z \subset \mathscr{R}\left[\mathscr{S}_{\left(t_{0}, T\right)} B\right]$, 并且存在单值线性胦射

$$
\mathscr{V} \in \mathscr{L}\left[Z \rightarrow L^{p}\left(t_{0}, T ; U\right)\right] \text {, }
$$

它使得 $z-\mathscr{S}_{\left(t_{0}, T\right)} B v, v-\mathscr{V} Z$, 此时 $k_{2}-\|\mathscr{V}\|_{\boldsymbol{Q}\left[Z \rightarrow L^{p}\left(t_{0}, T, v\right)\right]}$.

推论 1 设系统 (1) 满足假设 (L)' 以及(F), 则存在 $\alpha_{0}>0$, 只要 $0<\alpha<\alpha_{0}$, 系统 (1) 关于 $Z$ 中的元素具有精确能控性, 即 $Z \subset K_{\left(f_{0}, T\right)}(\xi ; \alpha F)$.

定理 2 设 $F-F(y),-A$ 是解析半群 $S(\cdot)$ 的生成算子. 如果系统 (1) 满足假设 (L)' 以及 (F), 则当 $\mathscr{R}[\mathscr{F}]$ 是 $L^{p}\left(t_{0}, T ; X\right)$ 中的有界集合时, 对任何 $\alpha>0$ 都有

$$
Z \subset K_{\left(t_{0}, T\right)}(\xi ; \alpha F) \text {. }
$$

证 设 $z \in Z, \xi \in X$ 以及 $\alpha>0$ 是任意给定的. 按照假设 (L)', 存在

$$
\bar{u} \in \mathscr{V}\left[z-S\left(T-t_{0}\right) \xi\right],
$$

并且可以定义一个把 $L^{p}\left(t_{0}, T ; U\right)$ 映入其自身的非线性算子 $\hat{\mathscr{Y}}$ :

$$
\hat{\mathscr{V}}_{u}=\mathscr{\mathscr { V }}\left[-\alpha \mathscr{S}_{\left(t_{0}, T\right)} \mathscr{F}(\vec{u}+u)\right], u(\cdot) \in L^{\mathscr{P}}\left(t_{0}, T ; U\right) .
$$

如果能解证明由 (10)式定义的算子 $\hat{\mathscr{V}}$ 存在不动点 $w(\cdot) \in L^{p}\left(t_{0}, T ; U\right)$, 即 $\hat{\mathscr{V}} w-w$, 则由 $\mathscr{Y}$ 的定义可知

$$
z-S\left(T-t_{0}\right) \xi-\alpha \mathscr{S}_{\left(t_{0}, T\right)} \mathscr{F}(\bar{u}+w)=\mathscr{P}_{\left(t_{0}, T\right)} B(\bar{u}+w),
$$

即 $z-y(T ; \bar{u}+w) \in K_{\left(t_{0}, T\right)}(\xi ; \alpha F)$, 于是 $Z \subset K_{\left(z_{0}, T\right)}(\xi ; \alpha F)$.

由于 $\mathscr{R}[\mathscr{F}]$ 在 $L^{P}\left(t_{0}, T ; X\right)$ 中是有界的,因此由

$$
\begin{aligned}
& \|(\hat{\mathscr{Y}} u)(\cdot)\|_{L^{p}\left(t_{0}, T, U\right)} \leqslant \alpha k_{1} k_{2}\|\mathscr{F}(\bar{u}+u)(\cdot)\|_{L^{p}\left(f_{0}, T, X\right)}, \\
& u(\cdot) \in L^{p}\left(t_{0}, T ; U\right),
\end{aligned}
$$

可知 $\hat{\mathscr{Y}}$ 把全空间 $L^{p}\left(t_{0}, T ; U\right)$ 映入它的一个有界子集. 可以证明,算子 $\hat{\mathscr{V}}$ 还是列的. 设 $\left\{u_{n}(\cdot) ; n-1,2, \cdots\right\}$ 是 $L^{p}\left(t_{0}, T ; U\right)$ 中弱收敛于 $u(\cdot) \in L^{p}\left(t_{0}, T ; U\right)$ 的控制序列. 类 似于文献 [4], 解映射 $y(\cdot ; \cdot)$ 把 $L^{p}\left(t_{0}, T ; U\right)$ 列紧地映人 $C\left(\left[t_{0}, T\right] ; X\right)$. 因此存在子序列 $\left\{U_{n_{m}}(\cdot) ; m-1,2, \cdots\right\}$ 使得在空间 $C\left(\left[t_{0}, T\right] ; X\right)$ 中 $\left\{\mathscr{F}\left(\bar{u}+u_{n_{m}}\right) ; m-1,2, \cdots\right\}$ 收 敛于 $(\bar{u}+u)$. 于是

$$
\lim _{m \rightarrow \infty}\left\|\mathscr{S}_{\left(t_{0}, T\right)} \mathscr{F}\left(\vec{u}+u_{n_{m}}\right)-\mathscr{S}_{\left(t_{0}, T\right)} \mathscr{F}(\bar{u}+u)\right\|_{Z}=0 .
$$

因为 $\mathscr{V} \in \mathscr{L}\left[Z \rightarrow L^{p}\left(t_{0}, T ; U\right)\right]$, 故在空间 $L^{p}\left(t_{0}, T ; U\right)$ 中有

$$
\begin{gathered}
\lim _{m \rightarrow \infty} \hat{\mathscr{V}} u_{n_{m}}-\lim _{m \rightarrow \infty} \mathscr{V}\left[-\alpha \mathscr{S}_{\left(t_{0}, T\right)} \mathscr{W}\left(\bar{u}+u_{n_{m}}\right)\right] \\
-\mathscr{V}\left[-\alpha \mathscr{S}_{\left(t_{0}, T\right)} \mathscr{F}(\bar{u}+u)\right]=\hat{\mathscr{V}} u .
\end{gathered}
$$

因此 $\hat{\mathscr{Y}}$ 把 $L^{p}\left(t_{0}, T ; U\right)$ 列紧地胦人它的一个有界子集. 根据 Shauder 不动点定理 ${ }^{[3]}$, 存在 $w(\cdot) \in L^{p}\left(t_{0}, T ; U\right)$ 使得 $\hat{\mathscr{V}} w=w$. 证毕.

假设 (N). 存在非线性函数 $F^{N}: X \times U \rightarrow X, N=1,2, \cdots$, (i) 它们关于 $N$ 一致地满 足 Lipschitz 条件(2), 即

$$
\begin{aligned}
& \left\|F^{N}\left(y_{1}, u_{1}\right)-F^{N}\left(y_{2}, u_{2}\right)\right\| \leqslant C_{1}\left\|y_{1}-y_{2}\right\|+C_{2}\left\|u_{1}-u_{2}\right\|_{U}, \\
& y_{1}, y_{2} \in X, u_{1}, u_{2} \in U, \quad N=1,2, \cdots ;
\end{aligned}
$$

(ii) 设 $t \in\left(t_{0}, T\right]$, 关于 $y(\cdot) \in C\left(\left[t_{0}, T\right] ; X\right), u(\cdot) \in L^{p}\left(t_{0}, T ; U\right)$ 一致地有

$$
\lim _{N \rightarrow \infty}\left\|\mathscr{S}_{\left(t_{0}, t\right)} F^{N}(y(\cdot), u(\cdot))-\mathscr{S}_{\left(t_{0}, t\right)} F(y(\cdot), u(\cdot))\right\|=0 .
$$

第 1 期

科学通报 
记 $y_{N}(\cdot ; u)$ 是以 $F^{N}$ 代替 (i) 中的 $F$ 所得的解快射, $\left(\mathbb{F}^{N} u\right)(\cdot)-F^{N}\left(y_{N}(\cdot ; u), u(\cdot)\right)$, $N-1,2, \cdots$.

定理 3 设系统 (1) 满足假设 $(\mathrm{L}) 、(\mathrm{~N})$ 以及用 $\mathscr{F}^{N}(N-1,2, \cdots)$ 代替 $\mathscr{F}^{-}$的假设 (P). 则存在 $a_{0}>0$, 只要 $0<\alpha<a_{0}$, 系统 (1) 都具有逼近能控性.

证设 $\alpha_{0}$ 由(8)式定义. 则当 $0<\alpha<\alpha_{0}$ 时, 对于 $N=1,2, \cdots$ 都有

$$
Z \subset \overline{K_{\left(t_{0}, T\right)}\left(\xi ; \alpha F^{N}\right)} \text {. }
$$

设 $z \in Z, \alpha>0$ 是任意给定的, 则当 $0<\alpha<\alpha_{0}$ 时都存在 $u_{s, N}(\cdot) \in L^{p}\left(t_{0}, T ; U\right)$ 使得

$$
\left\|z-y_{N}\left(T ; u_{s, N}\right)\right\|<\frac{\varepsilon}{2}, \quad N-1,2, \cdots .
$$

根据假设 (N) 可以证明, 存在正整数 $N(\varepsilon)$, 当 $N=N(\varepsilon), N(\varepsilon)+1, \cdots$ 时, 对任意的 $t \in\left(t_{0}\right.$, T] 都有

$$
\left\|y\left(t ; u_{\varepsilon, N}\right)-y_{N}\left(t ; u_{\varepsilon, N}\right)\right\| \leqslant \frac{\varepsilon}{2} e^{-M C_{1}(T-t)},
$$

特别地, 取 $t-T, N-N(\varepsilon)$ 则有 $\left\|z-y\left(T ; u_{\mathrm{s}, N(\varepsilon)}\right)\right\|<\varepsilon$.

谁论 2 设 $F-F(y),-A$ 是解析半群的生成算子. 如果系统(1)满足假设 (L)'、(N) 以及用 $\mathscr{F}^{N}$ 代替 $\mathscr{N}^{-}$的假设 $(\mathrm{F})$, 则当 $\mathscr{R}\left[\mathbb{F}^{N}\right](N-1,2, \cdots)$ 是 $L^{p}\left(t_{0}, T ; X\right)$ 中的有界集 ，合时,对任何 $\alpha>0$, 系统 (1) 都具有逼近能控性.

\section{* 齐 文}

[ 1 ] Henry, J., Thèse, Paris, 1978.

[ 2 ] Zhou H. X. (周㧶米), SIAM J. Control and Optimization, 21(1983), 551-565.

[ 3 ] -, Appl. Math. and Optimization, 8(1982), 275-285.

[ 4 ] Seidman, T. I. and Zhou H. X. (周㭤兴), SIAM J. Control and Optimization, 20(1982), 747-762.

[ 5 ] Smart, D. R., Fixed Point Theorems, Cambridge Univ., 1974. 